DOI: $10.15193 /$ zntj/2019/118/274

\author{
PIOTR ZARZYCKI, EMILIA SYKUT-DOMAŃSKA, ALDONA SOBOTA, \\ ANNA WIRKIJOWSKA
}

\title{
EFFECT OF WHOLEMEAL OAT FLOUR STORAGE ON CONTENT OF DIETARY FIBRE AND RHEOLOGICAL PROPERTIES
}

\begin{abstract}
Su m m a r y
The objective of the research study was to determine the effect of wholemeal oat flour storage on changes in a content of total (TDF), soluble (SDF), and insoluble (IDF) dietary fibre and in rheological properties of wholemeal oat flour. The analysis included the flours milled from naked oat grain in a laboratory. Those oat flours were stored for 3 months at temperatures of $20{ }^{\circ} \mathrm{C}, 4{ }^{\circ} \mathrm{C}$ and $-20{ }^{\circ} \mathrm{C}$. The apparent viscosity (initial, maximal, final, setback) was determined in $5 \%(\mathrm{w} / \mathrm{w})$ water-based suspensions. Regardless of the storage temperature, an increase was reported in the content of IDF as was a decrease in the content of SDF during the 3-month period of storing flour. The widest range of changes in the individual dietary fibre fractions was reported during storage at $20^{\circ} \mathrm{C}$. The changes in the rheological properties depended on the time and temperature of oat flour storage. The largest changes were reported in the case of the setback value during storage at $20^{\circ} \mathrm{C}$ and $4{ }^{\circ} \mathrm{C}$. The smallest range of changes in all the rheological properties was found in the flours stored at $-20^{\circ} \mathrm{C}$. During the 3 -month period of storing flour the range of changes in the rheological properties was inconsiderable and it did not depend on the contents of individual dietary fibre fractions, therefore it should not have any significant effect on the changes in the technological properties of flours. No unequivocal dependences were reported between the changes in the content of dietary fibre fractions and the rheological properties. During the entire 3-month storage period, the smallest changes occurred in the dietary fibre composition and rheological properties of oat flour during storage at the $-20^{\circ} \mathrm{C}$ temperature.
\end{abstract}

Key words: dietary fibre, oat flour, storage, rheological properties

\section{Introduction}

Owing to their high content of dietary fibre, mainly soluble fraction (SDF), oat products are commonly classified as food with highly beneficial physiological proper-

Dr inż. P. Zarzycki, dr inż. E. Sykut-Domańska, dr hab. A. Sobota, prof. UP, dr inż. A. Wirkijowska, Zakład Inżynierii i Technologii Zbóż, Katedra Technologii Surowców Pochodzenia Roślinnego i Gastronomii, Wydz. Nauk o Żywności i Biotechnologii, Uniwersytet Przyrodniczy w Lublinie, ul. Skromna 8, 20-704 Lublin. Kontakt: emilia.sykut-domanska@up.lublin.pl 
ties $[10,14]$. SDF stabilizes the level of glucose and contributes to the lowering of total cholesterol, cholesterol of low-density lipoprotein, cholesterol of very-low-density lipoprotein, and to the increase of cholesterol of high-density lipoprotein [2]. The primary mechanism responsible for this kind of physiological effect generated by SDF is its ability to increase the viscosity of the gastric content [8].

More and more often in the food production there are used flours with an increased content of dietary fibre. Research studies on the biochemical activity during storage and the impact of the fibre on the rheological properties of flour appear valuable for the food science. The research study on the changes in the viscosity during heating and, next, during cooling of gruels enables to determine swelling and grueling, shear strength and retrogradation [15]. The viscosity of oat flour pastes is determined by starch, $\beta$-glucans, lipids and protein [6].

During storage flour undergoes physical, chemical and biological processes, which can lead to changes in the content of dietary fibre and pasting properties. Antonini et al. [4] reported the increase in the $\beta$-glucan content during storage (12 months) of oat grain, possibly as a consequence of structural changes, which led to an increased extractability and solubility of $\beta$-glucan. Different results are presented by Åman et al. [3]. In the initial period of storage ( 0 - 6 month) significant decreases in the total and soluble mixed-linked $\beta$-glucan contents were found, probably as a result of the enzymatic activity ( $\beta$-glucanase) and the fragmentation of the $\beta$-glucan into smaller polymers, which, however, were not detected. During the remaining storage time (6 - 12 month) the contents of total mixed-linked $\beta$-glucan remained constant; this indicates no activity of the endogenous mixed-linked $\beta$-glucan degrading enzymes. Also Rakić et al. [13] reported a decrease in the $\beta$-glucan concentration during storage of oat grain. As regards pasting properties of flour, Brandolini et al. [7] reported a higher viscosity of flour gruels after storage compared to that of gruels from "fresh" flour.

In the reference data from the previous studies, there is a lack of information on the changes occurring in the dietary fibre fraction during storage of oat flour (at different temperatures) in relation to its pasting properties. Therefore a research study was undertaken to determine the effects of duration period (1, 2 and 3 months) and temperature $\left(20^{\circ} \mathrm{C}, 4^{\circ} \mathrm{C}\right.$ and $\left.-20{ }^{\circ} \mathrm{C}\right)$ of storage on the dietary fibre composition and pasting properties of wholemeal oat flour.

\section{Material and methods}

The experimental material consisted of wholemeal oat flours milled from 3 cultivars of naked oat grains ('Cacko', 'Siwek', 'Amant' from a plant breeding experimental station in Strzelce, Poland, 2016) in a laboratory (with the use of a Roller grinder type SK, Research Institute of the Bakery Industry, Poland). The oat flours were stored in glass containers at three different temperatures: $-20^{\circ} \mathrm{C}, 4^{\circ} \mathrm{C}$ and $20^{\circ} \mathrm{C}$, 
for $0,1,2$ and 3 months. The flour samples were produced and stored in duplicate. The control sample consisted of oat flour produced from three varieties of oat; this sample was not stored. The selection of oat varieties was made based on the content of dietary fibre therein. There were selected varieties with a similar content of total dietary fibre (TDF) and a different content of soluble (SDF) and insoluble fraction (IDF). The selection of oat varieties was made based on the pilot studies on 18 oat varieties.

The content of TDF, SDF and IDF was determined according to AOAC, 991.43 and AACC Method 32-07.01 [1, 5] in 3 replications for each flour.

The apparent viscosity of $5 \%(\mathrm{w} / \mathrm{w})$ suspensions of wholemeal oat flours in distilled water was determined immediately after milling (reference value), and after 1, 2 and 3 months of storage, according to Zarzycki and Sobota [15]. A rotary rheometer RM 180 (Mettler-Toledo AG, Switzerland, software RSI Orchestrator, ver. V6.5.8.), with coaxial cylinders without the bottom cylinder guard (shear rate of $1200 \mathrm{~s}^{-1}$ ), was utilized. The suspensions $(300 \mathrm{ml})$ were heated in a laboratory shaker (Elpin type 357, "Elpin Plus" s.c., Lubawa, Poland, amplitude: 3, frequency: $200 \mathrm{revs} / \mathrm{min}$ ) at a temperature ranging from 25 to $95{ }^{\circ} \mathrm{C}$; next they were kept at $95{ }^{\circ} \mathrm{C}$ for $20 \mathrm{~min}$, cooled to $50{ }^{\circ} \mathrm{C}$ and kept for $30 \mathrm{~min}$. A constant temperature gradient of $1{ }^{\circ} \mathrm{C} / \mathrm{min}$ was maintained during the heating and cooling processes. When the viscosity measuring agitation was stopped, the cylinders were immersed in the suspension and five consecutive readings were taken every 10 seconds. The measurements were made in 3 replications for each sample. The measurement of apparent viscosity included the initial viscosity at $25^{\circ} \mathrm{C}$, maximum viscosity while heating, final viscosity at $50^{\circ} \mathrm{C}$, final viscosity after $30 \mathrm{~min}$, and the setback.

The results were subjected to a statistical analysis (SAS ver. 9.2). The following was determined: mean values, standard deviations and the significance of differences between mean values (Duncan test, $\mathrm{p} \leq 0.05$ ). Additionally the correlation (Pearson, $\mathrm{p} \leq 0.05$ ) was determined between the pasting properties of wholemeal oat flour and the dietary fibre factions.

\section{Results and discussion}

The cultivar genotype of oat determines the dietary fibre composition and the pasting properties. As shown in the study, the response of individual oat cultivars to storage conditions can be reverse. For comparative purposes, the results obtained for individual oat varieties were given and the average values of the oat varieties investigated were calculated. Regardless of the storage temperature, an increase in the IDF and a decrease in the SDF were reported (Tab. 1). 
Table 1. Dietary fibre fractional composition of wholemeal oat flour during storage at different temperatures

Tabela 1. Skład frakcyjny błonnika pokarmowego pełnoziarnowej mąki owsianej przechowywanej w różnej temperaturze

\begin{tabular}{|c|c|c|c|c|c|}
\hline \multirow{2}{*}{$\begin{array}{c}\text { Storage temperature } \\
\text { Temperatura } \\
\text { przechowywania } \\
{\left[{ }^{\circ} \mathrm{C}\right]}\end{array}$} & \multirow{2}{*}{$\begin{array}{c}\text { Storage period } \\
{[\text { month] }} \\
\text { Czas przechowywania } \\
\text { [miesiąc] }\end{array}$} & \multicolumn{4}{|c|}{ Oat cultivar / Odmiana owsa } \\
\hline & & 'Cacko’ & 'Siwek’ & 'Amant' & $\overline{\mathrm{X}} * *$ \\
\hline \multicolumn{6}{|c|}{ TDF - Total dietary fibre [\% d.m.] / TDF - Błonnik pokarmowy całkowity [\% s.m.] } \\
\hline 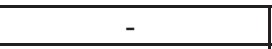 & $0 *$ & $17.7^{\mathrm{bc}} \pm 0.2$ & $17.40^{\mathrm{d}} \pm 0.10$ & $17.00^{\mathrm{f}} \pm 0.10$ & $17.4^{b} \pm 0.3$ \\
\hline \multirow{3}{*}{20} & 1 & $17.4^{\mathrm{c}} \pm 0.5$ & $15.6^{\mathrm{e}} \pm 0.2$ & $18.7^{\text {ed }} \pm 0.4$ & $17.3^{\mathrm{b}} \pm 1.4$ \\
\hline & 2 & $18.0^{\mathrm{bc}} \pm 0.7$ & $18.3^{\mathrm{c}} \pm 0.3$ & $23.9^{\mathrm{a}} \pm 0.9$ & $20.1^{\mathrm{a}} \pm 3.0$ \\
\hline & 3 & $19.9^{\mathrm{a}} \pm 0.2$ & $19.30^{\mathrm{ba}} \pm 0.10$ & $22.1^{b} \pm 0.2$ & $20.4^{\mathrm{a}} \pm 1.3$ \\
\hline \multirow{3}{*}{4} & 1 & $18.8^{\mathrm{ba}} \pm 0.4$ & $19.0^{b} \pm 0.3$ & $19.6^{\mathrm{cd}} \pm 0.2$ & $19.2^{\mathrm{a}} \pm 0.4$ \\
\hline & 2 & $17.80^{\mathrm{bc}} \pm 0.11$ & $19.2^{\mathrm{ba}} \pm 0.5$ & $20.9^{\mathrm{cb}} \pm 0.2$ & $19.3^{\mathrm{a}} \pm 1.4$ \\
\hline & 3 & $18.5^{\mathrm{bc}} \pm 1.0$ & $18.8^{\mathrm{bc}} \pm 0.4$ & $20.3^{\mathrm{c}} \pm 0.5$ & $19.2^{\mathrm{a}} \pm 1.0$ \\
\hline \multirow{3}{*}{-20} & 1 & $17.5^{\mathrm{c}} \pm 0.2$ & $17.0^{\mathrm{d}} \pm 0.2$ & $17.9^{\mathrm{ef}} \pm 0.3$ & $17.5^{\mathrm{b}} \pm 0.4$ \\
\hline & 2 & $17.60^{b c} \pm 0.12$ & $19.7^{\mathrm{a}} \pm 0.2$ & $20.6^{\mathrm{cb}} \pm 1.0$ & $19.3^{\mathrm{a}} \pm 1.4$ \\
\hline & 3 & $15.5^{\mathrm{d}} \pm 0.6$ & $15.7^{\mathrm{e}} \pm 0.4$ & $17.6^{\mathrm{ef}} \pm 1.3$ & $16.3^{\mathrm{b}} \pm 1.2$ \\
\hline \multicolumn{6}{|c|}{ SDF - Soluble dietary fibre [\% d.m.] / SDF - Błonnik pokarmowy rozpuszczalny [\% s.m.] } \\
\hline- & $0 *$ & $4.90^{\mathrm{a}} \pm 0.02$ & $6.30^{\mathrm{a}} \pm 0.03$ & $5.70^{\mathrm{a}} \pm 0.02$ & $5.7^{\mathrm{a}} \pm 0.7$ \\
\hline \multirow{3}{*}{20} & 1 & $4.00^{\mathrm{bc}} \pm 0.10$ & $4.50^{\mathrm{cd}} \pm 0.12$ & $4.6^{\mathrm{b}} \pm 0.4$ & $4.4^{\mathrm{b}} \pm 0.4$ \\
\hline & 2 & $4.20^{\mathrm{ba}} \pm 0.11$ & $4.4^{\mathrm{cd}} \pm 0.5$ & $4.40^{\mathrm{cb}} \pm 0.13$ & $4.3^{\mathrm{b}} \pm 0.3$ \\
\hline & 3 & $3.8^{\mathrm{bc}} \pm 0.3$ & $4.70^{\mathrm{cb}} \pm 0.10$ & $3.60^{\mathrm{d}} \pm 0.02$ & $4.0^{\mathrm{cb}} \pm 0.5$ \\
\hline \multirow{3}{*}{4} & 1 & $4.0^{b c} \pm 0.5$ & $5.2^{b} \pm 0.4$ & $4.70^{\mathrm{b}} \pm 0.11$ & $4.7^{b} \pm 0.6$ \\
\hline & 2 & $4.2^{\mathrm{ba}} \pm 0.6$ & $4.7^{\mathrm{cb}} \pm 0.4$ & $4.10^{\mathrm{cd}} \pm 0.12$ & $4.3^{b} \pm 0.5$ \\
\hline & 3 & $4.0^{b c} \pm 0.5$ & $5.0^{\mathrm{cb}} \pm 0.2$ & $4.60^{b} \pm 0.02$ & $4.5^{b} \pm 0.5$ \\
\hline \multirow{3}{*}{-20} & 1 & $4.11^{\mathrm{bac}} \pm 0.10$ & $5.0^{\mathrm{cb}} \pm 0.2$ & $4.6^{\mathrm{b}} \pm 0.2$ & $4.6^{b} \pm 0.4$ \\
\hline & 2 & $2.9^{\mathrm{d}} \pm 0.2$ & $3.91^{\mathrm{d}} \pm 0.11$ & $3.71^{\mathrm{d}} \pm 0.13$ & $3.5^{\mathrm{c}} \pm 0.5$ \\
\hline & 3 & $3.3^{\mathrm{dc}} \pm 0.2$ & $4.4^{\mathrm{cd}} \pm 0.3$ & $4.3^{\mathrm{cb}} \pm 0.4$ & $4.0^{\mathrm{cb}} \pm 0.6$ \\
\hline \multicolumn{6}{|c|}{ IDF - Insoluble dietary fibre [\% d.m.] / IDF - Błonnik pokarmowy nierozpuszczalny [\% s.m.] } \\
\hline- & $0^{*}$ & $12.8^{\mathrm{cd}} \pm 0.2$ & $11.10^{c} \pm 0.10$ & $11.31^{\mathrm{f}} \pm 0.12$ & $11.7^{\mathrm{c}} \pm 0.9$ \\
\hline \multirow{3}{*}{20} & 1 & $13.4^{\mathrm{bcd}} \pm 0.4$ & $11.10^{c} \pm 0.10$ & $14.1^{\mathrm{ed}} \pm 0.02$ & $12.9^{\mathrm{bc}} \pm 1.4$ \\
\hline & 2 & $13.8^{\mathrm{bc}} \pm 0.8$ & $13.9^{b} \pm 0.8$ & $19.4^{\mathrm{a}} \pm 0.9$ & $15.7^{\mathrm{a}} \pm 2.9$ \\
\hline & 3 & $16.1 \mathrm{a} \pm 0.6$ & $14.6^{\mathrm{b}} \pm 0.2$ & $18.51^{\mathrm{a}} \pm 0.12$ & $16.4^{\mathrm{a}} \pm 1.8$ \\
\hline \multirow{3}{*}{4} & 1 & $14.8^{\mathrm{ba}} \pm 0.9$ & $13.8^{b} \pm 0.6$ & $14.9^{\mathrm{cd}} \pm 0.2$ & $14.5^{\mathrm{ba}} \pm 0.8$ \\
\hline & 2 & $13.6^{\mathrm{bc}} \pm 0.5$ & $14.5^{b} \pm 0.9$ & $16.8^{\mathrm{b}} \pm 0.1$ & $15.0^{\mathrm{a}} \pm 1.6$ \\
\hline & 3 & $14.5^{\mathrm{b}} \pm 0.5$ & $13.8^{b} \pm 0.7$ & $15.7^{\mathrm{cb}} \pm 0.5$ & $14.7^{\mathrm{ba}} \pm 1.0$ \\
\hline \multirow{3}{*}{-20} & 1 & $13.4 b^{c d} \pm 0.3$ & $12.1^{\mathrm{c}} \pm 0.1$ & $13.2^{\mathrm{e}} \pm 0.5$ & $12.9^{\mathrm{bc}} \pm 0.7$ \\
\hline & 2 & $14.70^{\mathrm{b}} \pm 0.12$ & $15.80^{\mathrm{a}} \pm 0.02$ & $17.0^{b} \pm 0.9$ & $15.8^{\mathrm{a}} \pm 1.1$ \\
\hline & 3 & $12.1^{\mathrm{d}} \pm 0.8$ & $11.40^{\mathrm{c}} \pm 0.14$ & $13.3^{\mathrm{e}} \pm 0.9$ & $12.3^{\mathrm{c}} \pm 1.0$ \\
\hline
\end{tabular}

Explanatory notes / Objaśnienia:

* - non-stored flour (control sample) / mąka nieprzechowywana (próba kontrolna), $\overline{\mathrm{X}} * *$ - mean value of 3 oat cultivars: 'Cacko', 'Siwek', 'Amant' / wartość średnia z trzech odmian owsa: 'Cacko', 'Siwek', 'Amant'; d.m. - dry mater / s.m. - sucha masa. Table shows mean values \pm standard deviation / W tabeli przedstawiono wartości średnie \pm odchylenie standardowe; $\mathrm{n}=3$, a, b, $\mathrm{c}-$ mean values in columns and denoted by different letters differ statistically significantly $(\mathrm{p} \leq 0.05) /$ wartości średnie w kolumnach oznaczone różnymi literami różną się statystycznie istotnie $(\mathrm{p} \leq 0,05)$. 
The largest range of changes was reported during storage at $20{ }^{\circ} \mathrm{C}$. During the 3 -month storage a $40 \%$ increase (average of 3 oat cultivars) in the IDF was reported, a $17 \%$ increase in the TDF and a $30 \%$ decrease in the SDF. The highest changes in the dietary fibre compositions $\left(20^{\circ} \mathrm{C}\right)$ were found in 'Amant' (TDF: $30 \%$ increase; SDF: $37 \%$ decrease; IDF: $64 \%$ increase) and the lowest changes were found in 'Cacko' (TDF: $12 \%$ increase; SDF: $22 \%$ decrease; IDF: $26 \%$ increase). An increase in the content of TDF, IDF and a decrease in the content of SDF were also reported as regards the storage at $4{ }^{\circ} \mathrm{C}$; the average values were $10 \%, 26 \%$ and $21 \%$, respectively. With reference to IDF and TDF, the lowest range of changes: $5 \%$ and $6 \%$ on average, respectively, were reported during storage at $-20{ }^{\circ} \mathrm{C}$. Unlike the previous storage parameter $\left(20^{\circ} \mathrm{C}\right)$, a decrease in TDF was found. In the case of SDF, the range of changes $\left(30 \%\right.$ decrease) was similar to that found during storage at $20^{\circ} \mathrm{C}$. This fact indicated that the decrease reported in TDF at $-20^{\circ} \mathrm{C}$ was a consequence of lower changes in IDF.

The decrease in the SDF content in oat flour during storage confirms the result received by Åman et al. [3] and Rakić et al. [13]. This could be a result of the enzymatic depolymerisation of $(1,3)(1,4)-\beta-D$-glucans, which are the main components of SDF in oat [9]. The differences in the range of change in SDF during storage at different temperatures can be associated with the variability in the $\beta$-glucanase activity. The increase in IDF was probably caused by the decreasing availability of starch to enzyme hydrolysis on account of structural associations of amylose with other components such as lipids. The amylose molecules present in the oat starch are relatively short and they can easily form complexes with the lipids [6]. The oat flour lipid hydrolysis started immediately after milling [11]; it promoted the formation of amylose-free fatty acid complex.

The pasting properties of wholemeal oat flours are shown in Tab. 2 - 4. The viscosity profiles were similar for all the samples, with a hot paste viscosity at (or close to) $95^{\circ} \mathrm{C}$, a slight thinning phenomenon during the plateau at $95^{\circ} \mathrm{C}$ and an increase in the viscosity during cooling. The final viscosity was higher than the hot paste viscosity. During the cooling phase, complexes between the amylose and free fatty acids are formed, thus decreasing the amylose solubility and increasing the apparent viscosity of the paste [12]. The setback indicates the retrogradation ability, so this result may confirm the reduced ability of oat starch to retrograde. The highly branched amylopectin of oats starch is more active in the formation of the hot gel structure. Such a structure is less susceptible for retrogradation during cooling than that of the linear starch glucans [6]. 
Table 2. Apparent viscosity [mPa·s] of $5 \%$ gruels of wholemeal oat flour stored at $20{ }^{\circ} \mathrm{C}$

Tabela 2. Lepkość pozorna $[\mathrm{mPa} \cdot \mathrm{s}]$ 5-procentowych zawiesin pełnoziarnowej mąki owsianej przechowywanej w temp. $20^{\circ} \mathrm{C}$

\begin{tabular}{|c|c|c|c|c|c|}
\hline \multirow{2}{*}{$\begin{array}{l}\text { Storage period } \\
\text { [month] } \\
\text { Czas } \\
\text { składowania } \\
\text { [miesiąc] }\end{array}$} & \multirow{2}{*}{$\begin{array}{c}\text { Apparent viscosity } \\
\text { Lepkość pozorna }[\mathrm{mPa} \cdot \mathrm{s}]\end{array}$} & \multicolumn{4}{|c|}{ Oat cultivar / Odmiana owsa } \\
\hline & & 'Cacko' & 'Siwek’ & 'Amant' & $\overline{\mathrm{X}} * *$ \\
\hline \multirow{5}{*}{$0^{*}$} & $\begin{array}{l}\text { Initial at } 25^{\circ} \mathrm{C} \\
\text { Początkowa w temp. } 25^{\circ} \mathrm{C}\end{array}$ & $7.3^{\mathrm{e}} \pm 0.2$ & $7.5^{\mathrm{h}} \pm 0.1$ & $7.5^{\mathrm{j}} \pm 0.1$ & $7.4^{\mathrm{ba}} \pm 0.2$ \\
\hline & $\begin{array}{l}\text { Max while heating } \\
\text { Maksymalna w czasie } \\
\text { ogrzewania }\end{array}$ & $28.2^{\mathrm{c}} \pm 0.8$ & $32.4^{\mathrm{e}} \pm 1.1$ & $27.1^{\mathrm{f}} \pm 0.7$ & $29^{\mathrm{b}} \pm 3$ \\
\hline & $\begin{array}{l}\text { Final at } 50^{\circ} \mathrm{C} \\
\text { Końcowa w temp. } 50^{\circ} \mathrm{C}\end{array}$ & $35.2^{\mathrm{b}} \pm 0.8$ & $43.2^{\mathrm{bdc}} \pm 1.3$ & $39.9^{\text {edc }} \pm 0.9$ & $39.4^{\text {ed }} \pm 4.0$ \\
\hline & After $30 \mathrm{~min} /$ Po $30 \mathrm{~min}$ & $35.2^{\mathrm{b}} \pm 1.6$ & $41.9^{\mathrm{d}} \pm 1.2$ & $39.5^{\mathrm{ed}} \pm 0.3$ & $39^{b} \pm 3$ \\
\hline & Setback & $7.4^{\mathrm{e}} \pm 1.3$ & $11.7^{\mathrm{g}} \pm 3.4$ & $12.9^{\mathrm{hi}} \pm 0.2$ & $10.7^{\mathrm{ba}} \pm 3.0$ \\
\hline \multirow{5}{*}{1} & $\begin{array}{l}\text { Initial at } 25^{\circ} \mathrm{C} \\
\text { Początkowa w temp. } 25^{\circ} \mathrm{C}\end{array}$ & $7.6^{\mathrm{e}} \pm 0.1$ & $7.8^{\mathrm{h}} \pm 0.3$ & $7.5^{\mathrm{j}} \pm 0.1$ & $7.6^{\mathrm{a}} \pm 0.4$ \\
\hline & $\begin{array}{l}\text { Max while heating } \\
\text { Maksymalna w czasie } \\
\text { ogrzewania }\end{array}$ & $20.5^{\mathrm{d}} \pm 1.7$ & $22.4^{\mathrm{f}} \pm 1.4$ & $15.6^{\mathrm{hg}} \pm 1.1$ & $19^{\mathrm{c}} \pm 3$ \\
\hline & $\begin{array}{l}\text { Final at } 50^{\circ} \mathrm{C} \\
\text { Końcowa w temp. } 50^{\circ} \mathrm{C} \\
\end{array}$ & $40.3^{\mathrm{a}} \pm 3.7$ & $46 b^{\mathrm{ac}} \pm 1.1$ & $45.7^{\mathrm{a}} \pm 0.5$ & $44^{\mathrm{a}} \pm 3$ \\
\hline & $\begin{array}{l}\text { Final after } 30 \mathrm{~min} \\
\text { Końcowa po } 30 \mathrm{~min}\end{array}$ & $39.7^{\mathrm{a}} \pm 4.3$ & $46.2^{\mathrm{ba}} \pm 1.7$ & $44.1^{\mathrm{ba}} \pm 0.1$ & $43^{\mathrm{a}} \pm 4$ \\
\hline & Setback & $10.2^{\mathrm{e}} \pm 2.9$ & $14.2^{\mathrm{g}} \pm 0.1$ & $16.1^{\mathrm{g}} \pm 0.8$ & $13^{\mathrm{a}} \pm 3$ \\
\hline \multirow{5}{*}{2} & $\begin{array}{l}\text { Initial at } 25^{\circ} \mathrm{C} \\
\text { Początkowa w temp. } 25^{\circ} \mathrm{C}\end{array}$ & $7.3^{\mathrm{e}} \pm 0.2$ & $7.4^{\mathrm{h}} \pm 0.4$ & $7.4^{j} \pm 0.3$ & $7.4^{b} \pm 0.3$ \\
\hline & $\begin{array}{l}\text { Max while heating } \\
\text { Maksymalna w czasie } \\
\text { ogrzewania }\end{array}$ & $29.2^{\mathrm{c}} \pm 0.1$ & $31.3^{\mathrm{e}} \pm 1.2$ & $28.7^{\mathrm{f}} \pm 0.1$ & $30^{\mathrm{b}} \pm 1$ \\
\hline & $\begin{array}{l}\text { Final at } 50^{\circ} \mathrm{C} \\
\text { Końcowa w temp. } 50^{\circ} \mathrm{C}\end{array}$ & $41.2^{\mathrm{a}} \pm 1.8$ & $45^{\text {bdac }} \pm 2$ & $43.4^{\mathrm{ba}} \pm 0.5$ & $43^{\mathrm{a}} \pm 2$ \\
\hline & \begin{tabular}{|l|} 
Final after $30 \mathrm{~min}$ \\
Końcowa po $30 \mathrm{~min}$ \\
\end{tabular} & $40.2^{\mathrm{a}} \pm 1.8$ & $45^{\text {bdac }} \pm 3$ & $42.9^{\text {bac }} \pm 0.5$ & $43^{\mathrm{a}} \pm 3$ \\
\hline & Setback & $10.4^{\mathrm{e}} \pm 1.4$ & $11.8^{\mathrm{g}} \pm 1.9$ & $12.2^{i} \pm 0.5$ & $11^{\mathrm{ba}} \pm 1$ \\
\hline \multirow{5}{*}{3} & $\begin{array}{l}\text { Initial at } 25^{\circ} \mathrm{C} \\
\text { Początkowa w temp. } 25^{\circ} \mathrm{C}\end{array}$ & $6.9^{\mathrm{e}} \pm 0.1$ & $7.1^{\mathrm{h}} \pm 0.1$ & $6.8^{\mathrm{j}} \pm 0.1$ & $7.0^{\mathrm{d}} \pm 0.2$ \\
\hline & $\begin{array}{l}\text { Max while heating } \\
\text { Maksymalna w czasie } \\
\text { ogrzewania }\end{array}$ & $28.9^{c} \pm 1.0$ & $30.1^{\mathrm{e}} \pm 0.3$ & $27.9^{\mathrm{f}} \pm 0.2$ & $29^{b} \pm 1$ \\
\hline & $\begin{array}{l}\text { Final at } 50^{\circ} \mathrm{C} \\
\text { Końcowa w temp. } 50^{\circ} \mathrm{C}\end{array}$ & $39.5^{\mathrm{a}} \pm 0.2$ & $47.1^{\mathrm{a}} \pm 1.9$ & $41.0^{\mathrm{bdc}} \pm 3.0$ & $43^{\mathrm{ba}} \pm 4$ \\
\hline & $\begin{array}{l}\text { Final after } 30 \mathrm{~min} \\
\text { Końcowa po } 30 \mathrm{~min}\end{array}$ & $37.5^{\mathrm{ba}} \pm 0.2$ & $42.4^{\mathrm{dc}} \pm 1.0$ & $38.0^{\mathrm{e}} \pm 2.1$ & $39^{b} \pm 3$ \\
\hline & Setback & $7.8^{\mathrm{e}} \pm 1.6$ & $15.0^{\mathrm{g}} \pm 2.0$ & $11.0^{i} \pm 4.0$ & $8^{b} \pm 2$ \\
\hline
\end{tabular}

Explanatory notes as in Tab. 1. / Objaśnienia jak pod tab. 1. 
Table 3. Apparent viscosity [mPa.s] of $5 \%$ gruels of wholemeal oat flour stored at $4{ }^{\circ} \mathrm{C}$

Tabela 3. Lepkość pozorna $[\mathrm{mPa} \cdot \mathrm{s}] 5$-procentowych zawiesin pełnoziarnowej mąki owsianej przechowywanej w temp. $4{ }^{\circ} \mathrm{C}$

\begin{tabular}{|c|c|c|c|c|c|}
\hline \multirow{2}{*}{$\begin{array}{c}\text { Storage } \\
\text { period } \\
{[\text { month] }} \\
\text { Czas } \\
\text { składowania } \\
\text { [miesiąc] }\end{array}$} & \multirow{2}{*}{$\begin{array}{c}\text { Apparent viscosity } \\
\text { Lepkość pozorna }[\mathrm{mPa} \cdot \mathrm{s}]\end{array}$} & \multicolumn{4}{|c|}{ Oat cultivar / Odmiana owsa } \\
\hline & & ‘Cacko’' & 'Siwek' & 'Amant' & $\overline{\mathrm{X}} * *$ \\
\hline \multirow{5}{*}{$0^{*}$} & $\begin{array}{l}\text { Initial at } 25^{\circ} \mathrm{C} \\
\text { Początkowa w temp. } 25^{\circ} \mathrm{C}\end{array}$ & $7.3^{\mathrm{d}} \pm 0.2$ & $7.5^{\mathrm{e}} \pm 0.1$ & $7.5^{\mathrm{e}} \pm 0.1$ & $7.4^{\mathrm{ba}} \pm 0.2$ \\
\hline & $\begin{array}{l}\text { Max while heating } \\
\text { Maksymalna w czasie } \\
\text { ogrzewania }\end{array}$ & $28.2^{\mathrm{c}} \pm 0.8$ & $32.4^{\mathrm{c}} \pm 1.1$ & $27.1^{\mathrm{c}} \pm 0.7$ & $29.0^{\mathrm{b}} \pm 3.0$ \\
\hline & $\begin{array}{l}\text { Final at } 50^{\circ} \mathrm{C} \\
\text { Końcowa w temp. } 50^{\circ} \mathrm{C} \\
\end{array}$ & $35.2^{\mathrm{b}} \pm 0.8$ & $43.2^{\mathrm{ba}} \pm 1.3$ & $39.9^{b} \pm 0.9$ & $39.4^{\mathrm{ed}} \pm 4.0$ \\
\hline & After $30 \mathrm{~min} /$ Po $30 \mathrm{~min}$ & $35.2^{b} \pm 1.6$ & $41.9^{\mathrm{ba}} \pm 1.2$ & $39.5^{b} \pm 0.3$ & $39.0^{\mathrm{b}} \pm 3.0$ \\
\hline & Setback & $7.4^{\mathrm{d}} \pm 1.3$ & $11.7^{\text {ed }} \pm 3.4$ & $12.9^{\mathrm{d}} \pm 0.2$ & $10.7^{\mathrm{ba}} \pm 3.0$ \\
\hline \multirow{5}{*}{1} & $\begin{array}{l}\text { Initial at } 25^{\circ} \mathrm{C} \\
\text { Początkowa w temp. } 25^{\circ} \mathrm{C}\end{array}$ & $7.3^{\mathrm{d}} \pm 0.1$ & $7.6^{\mathrm{e}} \pm 0.6$ & $7.3^{\mathrm{e}} \pm 0.1$ & $7.4^{b} \pm 0.3$ \\
\hline & $\begin{array}{l}\text { Max while heating } \\
\text { Maksymalna w czasie } \\
\text { ogrzewania }\end{array}$ & $27.8^{c} \pm 0.1$ & $29.5^{\mathrm{c}} \pm 0.1$ & $28.8^{\mathrm{c}} \pm 0.8$ & $29.0^{\mathrm{b}} \pm 1.1$ \\
\hline & \begin{tabular}{|l|} 
Final at $50^{\circ} \mathrm{C}$ \\
Końcowa w temp. $50^{\circ} \mathrm{C}$ \\
\end{tabular} & $36.0^{b} \pm 0.3$ & $41.8^{\mathrm{ba}} \pm 1.4$ & $41.8^{\mathrm{ba}} \pm 0.5$ & $40^{\text {edc }} \pm 3$ \\
\hline & $\begin{array}{l}\text { Final after } 30 \mathrm{~min} \\
\text { Końcowa po } 30 \mathrm{~min}\end{array}$ & $35.4^{b} \pm 1.8$ & $40.3^{b} \pm 4.8$ & $40.1^{\mathrm{ba}} \pm 1.4$ & $39^{b} \pm 3$ \\
\hline & Setback & $7.8^{\mathrm{d}} \pm 1.4$ & $11.9^{\text {ed }} \pm 1.2$ & $13.5^{\mathrm{d}} \pm 0.7$ & $10^{\mathrm{ba}} \pm 2$ \\
\hline \multirow{5}{*}{2} & \begin{tabular}{|l|} 
Initial at $25^{\circ} \mathrm{C}$ \\
Początkowa w temp. $25^{\circ} \mathrm{C}$ \\
\end{tabular} & $7.2^{\mathrm{d}} \pm 0.2$ & $7.7^{\mathrm{e}} \pm 0.5$ & $7.2^{\mathrm{e}} \pm 0.1$ & $7.3^{\mathrm{b}} \pm 0.3$ \\
\hline & $\begin{array}{l}\text { Max while heating } \\
\text { Maksymalna w czasie } \\
\text { ogrzewania }\end{array}$ & $29.1^{c} \pm 0.3$ & $30.9^{c} \pm 0.7$ & $29.3^{c} \pm 1.6$ & $30.0^{\mathrm{b}} \pm 1.0$ \\
\hline & \begin{tabular}{|l|} 
Final at $50^{\circ} \mathrm{C}$ \\
Końcowa w temp. $50^{\circ} \mathrm{C}$ \\
\end{tabular} & $39.0^{\mathrm{a}} \pm 2.0$ & $45.0^{\mathrm{ba}} \pm 2.0$ & $42.2^{\mathrm{ba}} \pm 0.7$ & $42^{\mathrm{bac}} \pm 3$ \\
\hline & \begin{tabular}{|l|} 
Final after $30 \mathrm{~min}$ \\
Końcowa po $30 \mathrm{~min}$ \\
\end{tabular} & $36.7^{\mathrm{ba}} \pm 0.5$ & $43.3^{\mathrm{ba}} \pm 1.7$ & $39.0^{\mathrm{b}} \pm 2.0$ & $40^{b} \pm 3$ \\
\hline & Setback & $8.3^{\mathrm{d}} \pm 2.2$ & $13.1^{\mathrm{d}} \pm 0.9$ & $12.0^{\mathrm{d}} \pm 2.0$ & $9^{\text {ba }} \pm 3$ \\
\hline \multirow{5}{*}{3} & $\begin{array}{l}\text { Initial at } 25^{\circ} \mathrm{C} \\
\text { Początkowa w temp. } 25^{\circ} \mathrm{C}\end{array}$ & $7.4^{\mathrm{d}} \pm 0.2$ & $7.5^{\mathrm{e}} \pm 0.1$ & $7.4^{\mathrm{e}} \pm 0.3$ & $7.4^{\mathrm{b}} \pm 0.2$ \\
\hline & $\begin{array}{l}\text { Max while heating } \\
\text { Maksymalna w czasie } \\
\text { ogrzewania }\end{array}$ & $29.4^{\mathrm{c}} \pm 0.4$ & $32.0^{c} \pm 3.0$ & $28.2^{c} \pm 0.3$ & $30^{\mathrm{b}} \pm 2$ \\
\hline & \begin{tabular}{|l|} 
Final at $50^{\circ} \mathrm{C}$ \\
Końcowa w temp. $50^{\circ} \mathrm{C}$ \\
\end{tabular} & $38.6^{\mathrm{a}} \pm 1.3$ & $40.7^{b} \pm 0.9$ & $42.9^{\mathrm{a}} \pm 1.9$ & $41^{b d c} \pm 2$ \\
\hline & \begin{tabular}{|l|} 
Final after $30 \mathrm{~min}$ \\
Końcowa po $30 \mathrm{~min}$ \\
\end{tabular} & $39.0^{\mathrm{a}} \pm 0.7$ & $45.7^{\mathrm{a}} \pm 0.1$ & $42.8^{\mathrm{a}} \pm 3.1$ & $43^{\mathrm{a}} \pm 3$ \\
\hline & Setback & $6.9^{\mathrm{d}} \pm 2.1$ & $9.1^{\mathrm{ed}} \pm 3.2$ & $13.8^{\mathrm{d}} \pm 1.8$ & $12^{\mathrm{ba}} \pm 4$ \\
\hline
\end{tabular}

Explanatory notes as in Tab. 1. / Objaśnienia jak pod tab. 1. 
Table 4. Apparent viscosity [mPas $]$ of $5 \%$ gruels of wholemeal oat flour stored at $-20{ }^{\circ} \mathrm{C}$

Tabela 4. Lepkość pozorna $[\mathrm{mPa} \cdot \mathrm{s}]$ 5-procentowych zawiesin pełnoziarnowej mąki owsianej przechowywanej w temp. $-20^{\circ} \mathrm{C}$

\begin{tabular}{|c|c|c|c|c|c|}
\hline \multirow{2}{*}{$\begin{array}{c}\text { Storage period } \\
\text { [month] } \\
\text { Czas składowania } \\
\text { [miesiąc }]\end{array}$} & \multirow{2}{*}{$\begin{array}{c}\text { Apparent viscosity } \\
\text { Lepkość pozorna }[\mathrm{mPa} \cdot \mathrm{s}]\end{array}$} & \multicolumn{4}{|c|}{ Oat cultivar / Odmiana owsa } \\
\hline & & 'Cacko' & 'Siwek' & 'Amant' & $\overline{\mathrm{X}} * *$ \\
\hline \multirow{5}{*}{$0 *$} & $\begin{array}{l}\text { Initial at } 25^{\circ} \mathrm{C} \\
\text { Początkowa w temp. } 25^{\circ} \mathrm{C}\end{array}$ & $7.3^{\mathrm{e}} \pm 0.2$ & $7.5^{\mathrm{e}} \pm 0.1$ & $7.5^{\mathrm{e}} \pm 0.1$ & $7.4^{\mathrm{ba}} \pm 0.2$ \\
\hline & $\begin{array}{l}\text { Max while heating } \\
\text { Maksymalna w czasie } \\
\text { ogrzewania }\end{array}$ & $28.2^{\mathrm{d}} \pm 0.8$ & $32.4^{\mathrm{c}} \pm 1.1$ & $27.1^{\mathrm{c}} \pm 0.7$ & $29.0^{\mathrm{b}} \pm 3.0$ \\
\hline & $\begin{array}{l}\text { Final at } 50^{\circ} \mathrm{C} \\
\text { Końcowa w temp. } 50^{\circ} \mathrm{C}\end{array}$ & $35.2^{\mathrm{b}} \pm 0.8$ & $43.2^{\mathrm{a}} \pm 1.3$ & $39.9^{\mathrm{a}} \pm 0.9$ & $39.0^{\text {ed }} \pm 4.0$ \\
\hline & After $30 \mathrm{~min} /$ Po $30 \mathrm{~min}$ & $35.2^{b} \pm 1.6$ & $41.9^{\mathrm{ba}} \pm 1.2$ & $39.5^{\mathrm{a}} \pm 0.3$ & $39.4^{b} \pm 3.0$ \\
\hline & Setback & $7.4^{\mathrm{e}} \pm 1.3$ & $11.7^{\mathrm{d}} \pm 3.4$ & $12.9^{\mathrm{d}} \pm 0.2$ & $10.7^{\mathrm{ba}} \pm 3.0$ \\
\hline \multirow{5}{*}{1} & $\begin{array}{l}\text { Initial at } 25^{\circ} \mathrm{C} \\
\text { Początkowa w temp. } 25^{\circ} \mathrm{C}\end{array}$ & $7.1^{\mathrm{e}} \pm 0.2$ & $7.3^{\mathrm{e}} \pm 0.2$ & $7.0^{\mathrm{e}} \pm 0.1$ & $7.1^{\mathrm{c}} \pm 0.2$ \\
\hline & $\begin{array}{l}\text { Max while heating } \\
\text { Maksymalna w czasie } \\
\text { ogrzewania }\end{array}$ & $27.6^{\mathrm{d}} \pm 0.5$ & $29.9^{\mathrm{c}} \pm 0.2$ & $27.9^{\mathrm{cb}} \pm 1.9$ & $28.0^{\mathrm{b}} \pm 2.0$ \\
\hline & $\begin{array}{l}\text { Final at } 50^{\circ} \mathrm{C} \\
\text { Końcowa w temp. } 50^{\circ} \mathrm{C}\end{array}$ & $35.9 \mathrm{~b}^{\mathrm{a}} \pm 0.7$ & $38.4^{b} \pm 2.5$ & $40.7^{\mathrm{a}} \pm 0.3$ & $38.0^{\mathrm{e}} \pm 2.0$ \\
\hline & $\begin{array}{l}\text { Final after } 30 \mathrm{~min} \\
\text { Końcowa po } 30 \mathrm{~min}\end{array}$ & $35.6 \mathrm{~b}^{\mathrm{a}} \pm 1.1$ & $41.9^{\mathrm{ba}} \pm 1.5$ & $38.2^{\mathrm{a}} \pm 0.7$ & $39.0^{\mathrm{b}} \pm 3.0$ \\
\hline & Setback & $7.7^{\mathrm{e}} \pm 0.7$ & $9.2^{\mathrm{ed}} \pm 1.8$ & $12.9^{\mathrm{d}} \pm 0.1$ & $10.0^{\mathrm{ba}} \pm 3.0$ \\
\hline \multirow{5}{*}{2} & $\begin{array}{l}\text { Initial at } 25^{\circ} \mathrm{C} \\
\text { Początkowa w temp. } 25^{\circ} \mathrm{C}\end{array}$ & $7.4^{\mathrm{e}} \pm 0.2$ & $7.6^{\mathrm{e}} \pm 0.1$ & $7.3^{\mathrm{e}} \pm 0.1$ & $7.4^{\mathrm{ba}} \pm 0.2$ \\
\hline & $\begin{array}{l}\text { Max while heating } \\
\text { Maksymalna w czasie } \\
\text { ogrzewania }\end{array}$ & $31.2^{\mathrm{c}} \pm 0.4$ & $31.9^{\mathrm{c}} \pm 0.2$ & $30.9^{b} \pm 0.6$ & $31.0^{\mathrm{a}} \pm 1.0$ \\
\hline & $\begin{array}{l}\text { Final at } 50^{\circ} \mathrm{C} \\
\text { Końcowa w temp. } 50^{\circ} \mathrm{C}\end{array}$ & $37.6^{\mathrm{a}} \pm 1.2$ & $42.5^{\mathrm{a}} \pm 0.4$ & $40.0^{\mathrm{a}} \pm 3.0$ & $40.0^{\mathrm{edc}} \pm 3.0$ \\
\hline & $\begin{array}{l}\text { Final after } 30 \mathrm{~min} \\
\text { Końcowa po } 30 \mathrm{~min}\end{array}$ & $37.1^{\mathrm{ba}} \pm 1.3$ & $42.0^{\mathrm{ba}} \pm 3.0$ & $39.0^{\mathrm{a}} \pm 5.0$ & $39.0^{\mathrm{b}} \pm 3.0$ \\
\hline & Setback & $6.3^{\mathrm{e}} \pm 1.7$ & $8.4^{\mathrm{ed}} \pm 0.7$ & $9.1^{\mathrm{e}} \pm 2.7$ & $7.0^{b} \pm 1.0$ \\
\hline \multirow{5}{*}{3} & $\begin{array}{l}\text { Initial at } 25^{\circ} \mathrm{C} \\
\text { Początkowa w temp. } 25^{\circ} \mathrm{C}\end{array}$ & $6.8^{\mathrm{e}} \pm 0.1$ & $6.9^{\mathrm{e}} \pm 0.1$ & $6.7^{\mathrm{e}} \pm 0.1$ & $6.8^{\mathrm{d}} \pm 0.1$ \\
\hline & $\begin{array}{l}\text { Max while heating } \\
\text { Maksymalna w czasie } \\
\text { ogrzewania }\end{array}$ & $28.1^{\mathrm{d}} \pm 0.5$ & $29.9^{c} \pm 0.1$ & $28.5^{\mathrm{cb}} \pm 0.5$ & $29.0^{\mathrm{b}} \pm 1.0$ \\
\hline & $\begin{array}{l}\text { Final at } 50^{\circ} \mathrm{C} \\
\text { Końcowa w temp. } 50^{\circ} \mathrm{C} \\
\end{array}$ & $36.4^{\mathrm{ba}} \pm 1.4$ & $40.2^{\mathrm{ba}} \pm 2.3$ & $38.4^{\mathrm{a}} \pm 0.1$ & $38.0^{\mathrm{e}} \pm 2.0$ \\
\hline & $\begin{array}{l}\text { Final after } 30 \mathrm{~min} \\
\text { Końcowa po } 30 \mathrm{~min}\end{array}$ & $36.3^{\mathrm{ba}} \pm 1.1$ & $39.9^{\mathrm{ba}} \pm 1.3$ & $39.9^{\mathrm{a}} \pm 0.8$ & $39.0^{\mathrm{b}} \pm 2.0$ \\
\hline & Setback & $8.0^{\mathrm{e}} \pm 0.5$ & $10.2^{\mathrm{ed}} \pm 1.4$ & $10.0^{\text {ed }} \pm 0.3$ & $10.0^{\mathrm{ba}} \pm 2.0$ \\
\hline
\end{tabular}

Explanatory notes as in Tab. 1. / Objaśnienia jak pod tab. 1. 
Table 5. Correlation coefficients (Pearson) between dietary fibre content in wholemeal oat flour and apparent viscosity of $5 \%(\mathrm{w} / \mathrm{w})$ gruels of wholemeal oat flour during storage

Tabela 5. Współczynniki korelacji Pearsona pomiędzy zawartością błonnika pokarmowego w pełnoziarnowej mące owsianej a lepkością pozorną 5-procentowych $(\mathrm{m} / \mathrm{m})$ zawiesin pełnoziarnowej mąki owsianej w trakcie przechowywania

\begin{tabular}{|c|c|c|c|c|c|c|c|}
\hline \multirow{2}{*}{$\begin{array}{c}\text { Storage } \\
\text { temperature } \\
\text { Temperatura } \\
\text { składowania } \\
{\left[{ }^{\circ} \mathrm{C}\right]} \\
\end{array}$} & \multirow{2}{*}{\multicolumn{2}{|c|}{$\begin{array}{c}\text { Oat cultivar } \\
\text { Odmiana owsa }\end{array}$}} & \multicolumn{5}{|c|}{ Apparent viscosity / Lepkość pozorna } \\
\hline & & & $\begin{array}{l}\text { initial at } 25^{\circ} \mathrm{C} \\
\text { początkowa } \mathrm{W}\end{array}$ & $\begin{array}{l}\text { max during heating } \\
\text { maksymalna } \mathrm{w}\end{array}$ & $\begin{array}{l}\text { final at } 50^{\circ} \mathrm{C} \\
\text { końcowa } \mathrm{W}\end{array}$ & $\begin{array}{l}\text { final after } \\
30 \mathrm{~min}\end{array}$ & setback \\
\hline \multirow{12}{*}{20} & \multirow{4}{*}{ IDF } & 'Cacko' & -0.82 & 0.33 & 0.40 & 0.09 & -023 \\
\hline & & 'Siwek' & -0.83 & 0.39 & 0.57 & -0.22 & 0.27 \\
\hline & & 'Amant' & -0.64 & 0.40 & 0.24 & 0.01 & -0.54 \\
\hline & & $\overline{\mathrm{X}} * *$ & -0.76 & 0.38 & 0.53 & 0.06 & -0.54 \\
\hline & \multirow{4}{*}{ SDF } & 'Cacko' & 0.24 & 0.26 & -0.84 & -0.67 & -0.41 \\
\hline & & 'Siwek' & 0.02 & 0.48 & -0.77 & -0.68 & -0.48 \\
\hline & & 'Amant' & 0.86 & -0.09 & -0.31 & 0.15 & 0.39 \\
\hline & & $\overline{\mathrm{X}} * *$ & 0.45 & 0.18 & -0.88 & -0.44 & 0.13 \\
\hline & \multirow{4}{*}{ TDF } & 'Cacko' & -0.94 & 0.52 & 0.16 & -0.16 & -0.46 \\
\hline & & 'Siwek' & $-0.98 *$ & 0.75 & 0.23 & -0.67 & 0.04 \\
\hline & & 'Amant' & -0.55 & 0.46 & 0.21 & 0.05 & -0.55 \\
\hline & & $\overline{\mathrm{X}} * *$ & -0.81 & 0.6 & 0.32 & -0.11 & -0.65 \\
\hline \multirow{12}{*}{4} & \multirow{4}{*}{ IDF } & 'Cacko' & 0.28 & 0.02 & 0.28 & 0.39 & -0.15 \\
\hline & & 'Siwek' & 0.64 & -0.55 & -0.04 & 0.29 & 0.06 \\
\hline & & 'Amant' & $-0.96^{*}$ & 0.92 & 0.92 & 0.32 & -0.01 \\
\hline & & $\overline{\mathrm{X}} * *$ & -0.78 & 0.3 & 0.73 & 0.43 & 0.03 \\
\hline & \multirow{4}{*}{ SDF } & 'Cacko' & -0.11 & -0.37 & -0.65 & -0.59 & -0.02 \\
\hline & & 'Siwek' & -0.60 & 0.45 & 0.01 & -0.39 & -0.03 \\
\hline & & 'Amant' & $0.98^{*}$ & $-0.95 *$ & -0.85 & -0.19 & 0.14 \\
\hline & & $\overline{\mathrm{X}} * *$ & 0.81 & -0.41 & -0.81 & -0.47 & -0.01 \\
\hline & \multirow{4}{*}{ TDF } & 'Cacko' & 0.38 & -0.24 & -0.03 & 0.19 & -0.27 \\
\hline & & 'Siwek' & 0.67 & -0.63 & -0.06 & 0.20 & 0.10 \\
\hline & & 'Amant' & $-0.94 *$ & 0.90 & $0.94 *$ & 0.37 & 0.04 \\
\hline & & $\overline{\mathrm{X}} * *$ & -0.75 & 0.23 & 0.66 & 0.4 & 0.04 \\
\hline \multirow{12}{*}{-20} & \multirow{4}{*}{ IDF } & 'Cacko' & 0.76 & 0.80 & 0.67 & 0.55 & -0.92 \\
\hline & & 'Siwek' & 0.54 & 0.29 & 0.26 & 0.31 & -0.80 \\
\hline & & 'Amant' & -0.08 & $0.99 *$ & 0.32 & 0.05 & -0.81 \\
\hline & & $\overline{\mathrm{X}} * *$ & 0.36 & 0.87 & 0.66 & 0.84 & $-0.95 *$ \\
\hline & \multirow{4}{*}{ SDF } & 'Cacko' & 0.15 & -0.66 & -0.94 & $-0.97 *$ & 0.42 \\
\hline & & 'Siwek' & 0.23 & 0.41 & 0.28 & 0.37 & 0.86 \\
\hline & & 'Amant' & 0.44 & $-0.91 *$ & -0.06 & -0.07 & 0.85 \\
\hline & & $\overline{\mathrm{X}} * *$ & 0.30 & -0.54 & -0.15 & -0.48 & 0.72 \\
\hline & \multirow{4}{*}{ TDF } & 'Cacko' & 0.89 & 0.28 & -0.07 & -0.22 & -0.59 \\
\hline & & 'Siwek' & 0.86 & 0.64 & 0.53 & 0.65 & -0.52 \\
\hline & & 'Amant' & 0.13 & $0.97 *$ & 0.44 & 0.03 & -0.74 \\
\hline & & $\overline{\mathrm{X}} * *$ & 0.76 & 0.86 & 0.85 & 0.86 & -0.84 \\
\hline
\end{tabular}


Explanatory notes / Objaśnienia:

* - statistically significant correlation coefficient $(\mathrm{p} \leq 0.05)$ / współczynnik korelacji statystycznie istotny $(\mathrm{p} \leq 0,05)$. Other explanatory notes as in Tab. 1. / Pozostałe objaśnienia jak pod tab. 1.

While storing oat flours at $20{ }^{\circ} \mathrm{C}$ and $4{ }^{\circ} \mathrm{C}$, the largest changes of rheological properties, relative to the reference values, were reported for the setback; they were $23 \%$ and $16 \%$, respectively, at $20{ }^{\circ} \mathrm{C}$ and $4{ }^{\circ} \mathrm{C}$ (on average). For the initial and final viscosity, the range of changes did not exceed $10 \%$. During the storage at $-20{ }^{\circ} \mathrm{C}$, the largest changes $(8.5 \%)$ occurred in the initial viscosity (on average). With regard to other pasting properties, the range of changes was lower. This fact suggests that the 3 -month period of storage at various temperatures $\left(20^{\circ} \mathrm{C}, 4^{\circ} \mathrm{C}\right.$ and $\left.-20^{\circ} \mathrm{C}\right)$ seems to have not much effect on the changes in the apparent viscosity.

The depolymerization of $(1,3)(1,4)-\beta$-D-glucans may be partially responsible for the changes in the viscosity while storing oat flours. However the lack of any significant correlation between SDF and the paste viscosity implies that this is not the main cause of changes (Tab. 5). According to some research [16] freezing may influence the pasting properties of starch. However the authors' own research has proved relatively small changes in the rheological properties of oat flour after the 3-month storage period at a temperature of $-20^{\circ} \mathrm{C}$. A significant negative correlation was found between the changes in TDF and in the initial viscosity and also between the changes in IDF and in the initial viscosity. As shown in Tab. 5, in most cases, the correlations between changes in the fractions of fibre and in the pasting properties of oat flour were statistically insignificant (Person, $\mathrm{p} \leq 0.05$ ). Therefore it must be concluded that those processes are independent.

\section{Conclusions}

1. An increase in the content of IDF and a decrease in the content of SDF of wholemeal oat flour were reported after the 3-month period of storage. The range of changes was dependent on the oat cultivar. The cultivar genotype of oat determines the dietary fibre composition and the pasting properties. The largest range of changes in the dietary fibre fractions was reported during storage at $20^{\circ} \mathrm{C}$.

2. The measurements of pasting properties of wholemeal oat flour after milling and, next, after storage showed a small range of changes in the viscosity. This fact indicates that the 3-month storage of oat flour does not cause any deterioration in the rheological properties.

3. No unequivocal dependences were found between the changes in dietary fibre fraction and the rheological properties of oat flour gruels.

4. As regards the dietary fibre composition and the rheological properties of oat flour, the smallest changes occurred during the entire 3 -month storage period at $-20^{\circ} \mathrm{C}$. 


\section{References}

[1] AACC International: Approved Methods of Analysis. 11 ${ }^{\text {th }}$ ed. American Association of Cereal Chemistry International, St. Paul, Minnesota, USA, 2000.

[2] Alminger M., Eklund-Jonsson Ch.: Whole-grain cereal products based on a high-fibre barley or oat genotype lower post-prandial glucose and insulin responses in healthy humans. Eur. J. Nutr., 2008, 47, 294-300.

[3] Åman P., Graham H., Tilly A.-Ch.: Content and solubility of mixed-linked $(1 \rightarrow 3),(1 \rightarrow 4)-\beta-d-$ glucan in barley and oats during kernel development and storage. J. Cereal Sci., 1989, 10, 45-50.

[4] Antonini E., Lombardi F., Alfieri M., Diamantini G., Redaelli R., Ninfali P.: Nutritional characterization of naked and dehulled oat cultivar samples at harvest and after storage. J. Cereal Sci., 2016, 72, 46-53.

[5] AOAC: Official Methods of Analysis. $20^{\text {th }}$ ed. Association of Official Analytical Chemists International, Rockville, Maryland, USA, 2016.

[6] Berski W., Krystyjan M., Buksa K., Zięć G., Gambuś H.: Chemical, physical and rheological properties of oat flour affected by the isolation of beta-glucan preparation. J. Cereal Sci., 2014, 60, 533539.

[7] Brandolini A., Hidalgo A., Plizzari L.: Storage-induced changes in einkorn (Triticum monococcum L.) and breadwheat (Triticum aestivum L. ssp. aestivum) flours. J. Cereal Sci., 2010, 51, 205-212.

[8] Dikeman Ch.L., Fahey G.C.Jr.: Viscosity as related to dietary fibre: A review. Crit. Rev. Food Sci. Nutr., 2006, 46, 649-663.

[9] Flander L., Salmenkallio-Marttila M., Suortti T., Autio K.: Optimization of ingredients and baking process for improved wholemeal oat bread quality. LWT, 2007, 40, 860-870.

[10] Kołodziejczyk P., Michniewicz J.: Ziarno zbóż i produkty zbożowe jako źródła błonnika pokarmowego. Żywność. Nauka. Technologia. Jakość, 2018, 25, 3 (116), 5-22.

[11] Lampi A.M., Damerau A., Li J., Moisio T., Partanen R., Forssell P., Piironen V.: Changes in lipids and volatile compounds of oat flours and extrudates during processing and storage. J. Cereal Sci., 2015, 62, 102-109.

[12] Mestres C., Nago M., Akissoe N., Matencio F.: End use quality of some African corn kernels. 2. Cooking behavior of whole dry-milled flours; incidence of storage. J. Agric. Food Chem., 1997, 45, 565-571.

[13] Rakić S., Janković S., Marčetić M., Živković D., Kuzevski J.: The impact of storage on the primary and secondary metabolites, antioxidant activity and digestibility of oat grains (Avena sativa). J. Funct. Foods, 2014, 7, 373-380.

[14] Sangwan S., Singh R., Tomar S.K.: Nutritional and functional properties of oats: An update. J. Innov. Biol., 2014, 1, 3-14.

[15] Zarzycki P., Sobota A.: Effect of storage temperature on falling number and apparent viscosity of gruels from wheat flours. J. Food Sci. Technol., 2015, 52, 437-443.

[16] Zhang Y., Simsek S.: Physicochemical changes of starch in refrigerated dough during storage. Carbohydr. Polym., 2009, 78, 268-274.

\section{WPLYW PRZECHOWYWANIA PELNOZIARNOWEJ MĄKI OWSIANEJ NA ZAWARTOŚĆ BLONNIKA POKARMOWEGO I WLAŚCIWOŚCI REOLOGICZNE}

Streszczenie

Celem pracy było określenie wpływu przechowywania mąki razowej owsianej na zmiany zawartości błonnika pokarmowego całkowitego (TDF), frakcji rozpuszczalnej (SDF) i nierozpuszczalnej (IDF) oraz właściwości reologicznych mąki. Badaniom poddano mąki otrzymane w przemiale laboratoryjnym ziarna owsa nagiego. Mąki owsiane przechowywano przez 3 miesiące w temp. $20{ }^{\circ} \mathrm{C}, 4^{\circ} \mathrm{C}$ i $-20^{\circ} \mathrm{C}$. Lep- 
kość pozorną (początkową, maksymalną, końcową oraz setback) określano w 5-procentowych (m/m) wodnych zawiesinach. Niezależnie od temperatury odnotowano wzrost zawartości IDF oraz zmniejszenie zawartości SDF w trakcie 3-miesięcznego przechowywania mąki. Największy zakres zmian występujący $\mathrm{w}$ poszczególnych frakcjach błonnika odnotowano w czasie przechowywania w temp. $20{ }^{\circ} \mathrm{C}$. Zmiany właściwości reologicznych były zależne od czasu i temperatury przechowywania mąki owsianej. Największe zmiany wystąpiły w przypadku wartości setback, w trakcie przechowywania $\mathrm{w}$ temp. $20^{\circ} \mathrm{C}$ i $4{ }^{\circ} \mathrm{C}$. Najmniejszy zakres zmian wszystkich badanych właściwości reologicznych stwierdzono w mąkach przechowywanych $\mathrm{w}$ temp. $-20{ }^{\circ} \mathrm{C}$. Obserwowany zakres zmian właściwości reologicznych w trakcie 3miesięcznego przechowywania mąki, niezależnie od zawartości poszczególnych frakcji błonnika pokarmowego, był niewielki i nie powinien istotnie wpływać na zmiany właściwości technologicznych mąki. Nie odnotowano jednoznacznych zależności pomiędzy zmianami zawartości frakcji błonnika a badanymi właściwościami reologicznymi. Najmniejsze zmiany w ciągu 3-miesięcznego przechowywania w odniesieniu do składu błonnika pokarmowego oraz właściwości reologicznych mąki owsianej wystąpiły podczas przechowywania w temp. $-20^{\circ} \mathrm{C}$.

Słowa kluczowe: błonnik pokarmowy, mąka owsiana, przechowywanie, właściwości reologiczne 